\title{
A cluster of Legionnaires' disease caused by Legionella longbeachae linked to potting compost in Scotland, 2008-2009
}

S J Pravinkumar (Josephine.Pravinkumar@lanarkshire.scot.nhs.uk) ${ }^{1}$, G Edwards ${ }^{2}$, D Lindsay ${ }^{2}$, S Redmond Re J Stirling $^{3}$, R House $^{4}$,

J Kerr ${ }^{1}$, E Anderson 5 , D Breen ${ }^{6}$, 0 Blatchford ${ }^{4}$, E McDonald $^{4}$, A Brown $^{2}$

1. National Health Service Lanarkshire, Hamilton, United Kingdom

2. Scottish Legionella Reference Laboratory, Stobhill Hospital, Glasgow, United Kingdom

3. South Lanarkshire Council, Atholl House, East Kilbride, United Kingdom

4. Health Protection Scotland, Clifton House, Glasgow, United Kingdom

5. Greater Glasgow and Clyde Health Board, Dalian House, Glasgow, United Kingdom

6. National Health Service Dumfries and Galloway, Crichton Hall, Dumfries, United Kingdom

Citation style for this article:

Citation style for this article: Pravinkumar SJ, Edwards G, Lindsay D, Redmond S, Stirling J, House R, Kerr J, Anderson E, Breen D, Blatchford O, McDonald E, Brown A. A cluster of Legionnaires' disease caused by Legionella longbeachae linked to potting compost in Scotland, 2008-2009. Euro Surveill. $2010 ; 15$ (8):pii=19496.

Available online: http://www.eurosurveillance.org/ViewArticle.aspx?Articleld=19496

This article has been published on 25 February 2010

Three cases of Legionnaires' disease caused by Legionella longbeachae Sg 1 associated with potting compost have been reported in Scotland between 2008 and 2009. The exact method of transmission is still not fully understood as Legionnaires' disease is thought to be acquired by droplet inhalation. The linked cases associated with compost exposure call for an introduction of compost labelling, as is already in place in other countries where $L$. longbeachae outbreaks have been reported.

Legionella longbeachae has been rarely detected as the cause of respiratory illness in Scotland. Five cases have been reported in Scotland between 1995 and 2009 (Health Protection Scotland: personal communication) However, it is a well recognised type in Australia and New Zealand, where cases have been epidemiologically associated with the use of potting compost. $[1,2]$

\section{Case Reports}

Case 1 was notified in 2008, admitted to hospital and was severely unwell for a number of months before recovering slowly. Case 2 became unwell in 2009 and was admitted to hospital with respiratory and gastrointestinal symptoms, but died as a result of multiple organ failure. There was no history of travel or occupational exposure. No significant environmental exposure to cooling towers or aerosol producers was identified. The patients' domestic water systems were sampled, but no Legionella were detected. Case 1 had recently begun growing tomatoes in a conservatory attached to the house, using potting compost for this purpose. Case 2 was a keen gardener and had spent long periods of time in the greenhouse and had used potting compost to pot plants.

Case 3 was identified in 2009 and required hospitalisation as well. Again, there was no history of foreign travel and no significant exposure to cooling towers or aerosol producers in the vicinity. The case had been using compost to plant bulbs in pots in their garden.

The three cases had a median age of 65 years (range: 58-65 years) and were treated for community-acquired pneumonia. Cases 1 and 2 had risk factors such as smoking and underlying medical conditions for developing Legionnaires' disease.

\section{Laboratory results}

L. longbeachae Sg 1 was isolated from the first two patients. The third patient had a very high antibody titre of 1:8,192 against L. longbeachae Sg 1 by immunofluorescent antibody (IFA) testing [3]. Four isolates of L. longbeachae Sg 1 were obtained from the implicated potting composts ranging in counts from 4,000 $\mathrm{cfu} / \mathrm{g}$ to $80,000 \mathrm{cfu} / \mathrm{g}$. The identity of the Legionella species was confirmed by IFA and mip speciation (http:// www.hpa-bioinformatics.org.uk/cgi-bin/legionella/ mip/mip_id.cgi). Patient and environmental isolates were genotyped by amplified fragment length polymorphism (AFLP) as recommended by the European Working Group for Legionella Infections (EWGLI), although restriction fragment length polymorphism (RFLP) and pulsed-field gel electrophoresis (PFGE) [4] have been used in the past. We identified two AFLP types that we called $A$ and $B$ as there is currently no nomenclature from $L$. longbeachae AFLP types. Case 1 had AFLP A and the implicated compost contained both AFLP A and B. Case 2 had AFLP type B and the implicated compost contained AFLP type B. One of the compost types used by Cases 1 and 2 were of the same brand. There was no patient isolate from Case 3 but isolate from the implicated compost contained AFLP type A similar to the strain that had infected Case 1. Case 3 had used a brand of compost different from the one used by Cases 1 and 2. 
The potting composts implicated in the first two cases was composed of shredded green waste that is heattreated at above $65{ }^{\circ} \mathrm{C}$ for five to 10 days and of 30-50\% peat, which is not heat-treated. A second type of compost used by Case 2 was composed of composted bark, green material and not heat-treated $75-80 \%$ peat. The compost involved in the third case was a bulb booster compost made from expanded wood fibre, coir, and bark. All the types of compost used conformed to the 'PAS 100 Standard' (the British Standards Institution's Publicly Available Specification for composted material, which outlines the minimum requirements for the process of composting, the selection of materials from which compost is made and how it is labelled [5]).

\section{Public health measures}

Following the detection of Case 1 , a report highlighting the association with compost was prepared by the local National Health Service board consultant in public health medicine and was submitted through the department of consumer and trading standards of the local authority to the United Kingdom's (UK) Department of Business Innovations and Skills (BIS), which leads the regulatory reform agenda across the UK government. The report was sent to BIS with a view to consider statutory changes in terms of compost labelling and recommended that, although the incidence of $L$. longbeachae is rare in Scotland, a review of compost labelling with regards to L. longbeachae could be considered in the UK.

The family of Case 2 were advised against use of the greenhouse and to avoid any contact with the plants which have been re-potted using the compost under investigation. The greenhouse had to be cleared and given a chemical wash out prior to re-use. The decontamination of the greenhouse was carried out as a precautionary measure and follow-up ground soil samples were negative for Legionella.

For Case 3, given that the infection was likely acquired from contact with the compost in an open garden and that no other person in the house was involved in gardening, no public health control measures were deemed to be required.

\section{Discussion}

This is the first incident of two linked cases of $L$. longbeachae Sg 1 reported in Scotland. A common source was implicated and the cases were linked epidemiologically in terms of time, place and mechanism of exposure. The third case, although not directly linked, showed that the infection was not necessarily specific to a particular brand of compost. Nevertheless, the occurrence of the linked cases associated with compost exposure calls for the introduction of compost labelling as is already in place in other countries where L. longbeachae outbreaks have been reported. $[6,7]$.

It has been reported that various Legionella strains have been isolated from different types of potting soils including peat [8]. In Australia, where cases and outbreaks of $L$. longbeachae have been reported, the standards for composts, soil conditioners and mulches provide clear guidance to commercial producers of compost on how to process organic materials into compost in a safe and effective way [7]. These standards also include requirements for labelling bags and promoting safe and healthy gardening practices. Public health advice includes the risk of Legionnaires' disease following exposure to compost or potting soil.

A recent article by Casati et al. [8] has also highlighted that potting soils are an alternative and important, but probably underestimated, source of Legionella infection, not only by L. longbeachae but also by other Legionella species known to cause Legionnaires' disease. The article recommends collecting environmental samples, in particular potting soils, in addition to water samples as part of environmental investigations following a case of Legionnaires' disease.

A case control study by Connor et al. [9] demonstrated a significant risk of acquiring $L$. longbeachae infection (odds ratio 4.74, 95\% confidence interval: 1.65-13.55, $\mathrm{P}=0.004$ ) associated with recent use of potting mix. The study also showed that awareness of a possible health risk with potting mix protected against illness.

Casati et al. highlighted that although contamination of soil by Legionella was until recently considered to be limited to Australia, an association between cases of Legionnaires' disease and gardening or use of potting mixes has been identified in Japan, the United States, the Netherlands and Switzerland [8]. In the UK, only nine cases of $L$. longbeachae have been reported since 1984 (Health Protection Agency, personal communication). Five of them, including the three cases in this report, occurred in Scotland. Although uncommon, our experience, and reports in other parts of the world as highlighted above indicate that Legionnaires' disease can be acquired after contact with contaminated potting soil and is not limited to aerosolisation of contaminated water.

The current guidance on the management of Legionella incidents, outbreaks and clusters in the community, which has been published by the Health Protection Network [10] includes a hypotheses-generating questionnaire that explores the possibility of exposure to compost, gardening and pressure hoses. It is essential that the initial risk assessment at the time of clinical management of cases includes the risk of exposure to these factors to guide investigations.

On a cautionary note, current urinary antigen tests will not detect $L$. longbeachae infection and therefore cannot be relied upon by laboratories to make a diagnosis. Therefore, in the event of a community-acquired pneumonia with no discernible cause, serum and respiratory secretions should be sent to a national Legionella 
reference laboratory for PCR and culture, particularly if the case has a connection to gardening.

The cases reported here emphasise the need for a voluntary use in the UK of an industry-agreed warning label for potting soil, as the risk of Legionnaires' disease associated with compost is now clearly identified.

\section{Acknowledgements}

We would like to acknowledge the help of Eddie Docherty and Catherine Keachie, NHS Lanarkshire and Francis Shields, South Lanarkshire Council.

\section{References}

1. Grove DI, Lawson PJ, Burgess JS, Moran JL, O'Fathartaigh MS Winslow WE. An outbreak of Legionella longbeachae infection in an intensive care unit? J Hosp Infect. 2002;52(4):250-8.

2. Cramp GJ, Harte D, Douglas NM, Graham F, Schousboe M, Sykes K. An outbreak of Pontiac fever due to Legionella longbeachae serogroup 2 found in potting mix in a horticultural nursery in New Zealand. Epidemiol Infect. 2010;138(1):15-20. Epub 2009 Sep 28

3. Fallon RJ, Abraham WH. Polyvalent heat-killed antigen for the diagnosis of infection with Legionella pneumophila J Clin Pathol. 1982;35(4):434-8.

4. Montanaro-Punzengruber JC, Hicks L, Meyer W, Gilbert GL. Australian Isolates of Legionella longbeachae Are Not a Clonal Population. Journal of J Clin Microbiol. 1999;37(10):3249-54.

5. Compost specifications for different applications. Banbury: WRAP [Internet]. Available from: http://www.wrap.org.uk/ composting/compost_specifications/index.html. [Accessed 25 Feb 2010].

6. Broadbent C. Guidance for the Control of Legionella. National Environmental Health Forum Monographs. Water Series No. 1. South Australian Health Commission for the National Environmental Health Forum; 1996.

7. NSW Health. NSW Code of Practice for the Control of Legionnaires' disease, 2nd ed. North Sydney: New South Wales Department of Health; June 2004.

8. Casati S, Gioria-Martinoni A, Gaia V. Commercial potting soils as an alternative infection source of Legionella pneumophila and other Legionella species in Switzerland. Clin Microbiol Infect. 2009;15(6):571-5.

9. O'Connor BA, Carman J, Eckert K, Tucker G, Givney R, Cameron S. Does using potting mix make you sick? Results from a Legionella longbeachae case-control study in South Australia. Epidemiol Infect. 2007;135(1):34-9.

10. Health Protection Network. Guideline on Management of Legionella Incidents, Outbreaks and Clusters in the Community. Health Protection Network Scottish Guidance 2. Glasgow: Health Protection Scotland; 2009. 